\title{
Ultra-pulsed Carbon Dioxide Laser for the Treatment of Melanocytic Nevi
}

Ali Mohamed Gargoom *, Gamal Ahmed Duweb

Dermatology Department, Faculty of Medicine, Benghazi University. Benghazi-Libya

*Corresponding Author : Ali Mohamed Gargoom, Dermatology Department, Faculty of Medicine, Benghazi University. Benghazi-Libya; E-mail: gargoom@yahoo.com

Received date : July 02, 2019; Accepted date : July 20, 2019; Published date : July 24, 2019

Citation : Gargoom AM, Duweb GA (2019) Ultra-pulsed Carbon Dioxide Laser for the Treatment of Melanocytic Nevi. J Dermatol Dermatit DOI: 10.31579/2578-8949/054

Copyright : @ 2019 Gargoom AM. This is an open-access article distributed under the terms of The Creative Commons Attribution License, which permits unrestricted use, distribution, and reproduction in any medium, provided the original author and source are credited.

\section{Abstract}

Congenital melanocytic nevi (CMN) even small-sized as well as acquired melanocytic nevi (AMN) are of common cosmetic concerns and getting rid of them remain challenging. The aim of this study is to evaluate the efficacy and safety of the ultra-pulsed CO2 laser for the treatment of melanocytic nevi. 15 male and female Libyan patients with 16 AMN and 7 CMN were treated with multiple sessions of ultra-pulsed $\mathrm{CO} 2$ laser at 1-month intervals. Clinical response at 6 months after final treatment was graded as poor $(<50 \%)$, moderate $(50-75 \%)$, good $(>75 \%)$ and excellent where the skin of the lesion returned normal as the rest of the surrounding skin. All treated nevi showed good to excellent cosmetic outcomes after 2 or 4 treatment sessions. The post laser complication were minimal and transit except for one atrophic scar. No recurrence of pigmentation during the six months of follow-up period.

Conclusion: Ultra-pulsed $\mathrm{CO} 2$ laser seems to be effective and associate with minimum adverse side effect in the treatment of small CMN and AMN.

Keywords: UCO2, laser, melanocytic, nevus, acquired, congenital

\section{Introduction}

Melanocytic nevi may present at birth in about $1 \%-6 \%$ of newborn infants and referred as congenital melanocytic nevi (CMN) or may developed later on in life where known as acquired melanocytic nevi $(\mathrm{AMN})[1,2]$. Depending on the clinical examination solely, sometimes it is hardly possible to differentiate between congenital and acquired nevi and documentation since birth may be the only way to prove the congenital origin [3].

Congenital melanocytic nevi classified according to their surface areas into; small $\mathrm{CMN}$ with a diameter $<1.5 \mathrm{~cm}$, medium-sized CMN measure between 1.5 and $19.5 \mathrm{~cm}$ and large CMN with diameters > $19.5 \mathrm{~cm}[2,4]$. Acquired melanocytic nevi are benign proliferations of melanocytic cells

(Also known as nevus cells) that are characterized histopathologically by presence of nests of nevus cells in the dermo-epidermal junction (junctional nevi), or in the papillary dermis (compound nevi) or deep in the dermis (dermal nevi) [5].

Different modality of treatment were tried to treat melanocytic nevi including; surgical excision, cryotherapy, dermabrasion, curettage and chemical peeling. Although some of these modality of treatment is effective in removing the nevi but the risk of dyschromia and scar formation limit their uses. Nevertheless, nevi in esthetically sensitive or surgically challenging areas and large nevi is another important obstacle. In these conditions laser treatment of melanocytic nevi is the appropriate alternative choice $[5,6]$. Both pigment-specific alexandrite $(\mathrm{Al}, 755 \mathrm{~nm})$, neodymium: yttrium aluminum-garnet $(\mathrm{Nd}: Y A G, 1064$ $\mathrm{nm}$ ) and frequency-doubled Nd:YAG laser ( fd Nd:YAG, $532 \mathrm{~nm}$ ) and ablating lasers such as carbon dioxide (CO2) and erbium: yttriumaluminum-garnet (Er:YAG) and in combination with each other, have been found to be effective in the treatment of melanocytic nevi but with variable outcome and sequels [5-8].

Histological examination after various laser procedure reveals persistence of nevomelanocytic cells in the papillary dermis which increase the possibility of recurrence. For this reason, long-pulsed pigment-specific laser and ablating laser are more efficient [5].

Carbon dioxide laser considered as the gold standard in ablative lasers with its wavelength $(10.600 \mathrm{~nm})$ which is selectively absorbed by water.
Since approximately $77 \%$ of skin consists of water, this absorption was the optimal condition for the ablation effect. The major drawback of $\mathrm{CO} 2$ laser in the continuous mode is considerable possibility of texture changes because of its uncontrollable penetration depth [5]. The emergence of the superpulsed $\mathrm{CO} 2$ laser and more recently the ultrapulsed $\mathrm{CO} 2$ (UCO2) laser which delivers high energy beam in very short impulses minimizes the risks of scarring [8,9]. The present work was designed to evaluate the efficiency and safety of UCO2 laser ablation in the treatment of melanocytic nevi in a group of patients with skin type IV and V.

\section{Material and Methods}

Between January 2016 and October 2017, fifteen male and female Libyan patients aged between 15 years to 35 years (mean age 19.3 years) with a total of 23 clinically benign-appearing melanocytic nevi were enrolled in the present study. Sixteen out of the 23 studied nevi were AMN and seven were CMN. Melanocytic nevi with a diameter of $3 \mathrm{~cm}$ or larger or with variable color, irregular border, and asymmetry were excluded. Melanocytic nevi with sudden increase in size or textural changes were also excluded. All included patients had Fitzpatrick's skin types IV and V. Pregnant or lactating female or patients with keloid tendency or family history of melanoma were excluded.

Laser procedure, the potential complications and the expected outcomes were explained to every patient prior to laser session. An informed consent was obtained from each patient. The lesions were cleaned with disinfecting solution then infiltrated with $2 \%$ Lignocaine via insulin syringe attached to $29-\mathrm{G}$ needle. Lesions were treated with UCO2 laser (Fraxis from ilooda; $10,600 \mathrm{~nm} ; 100-200 \mathrm{~Hz} ; 200-400 \mu$ s) with fixed spotsize handpiece (spot diameter $100 \mathrm{~mm}$ ). The photoablated tissue was gently removed with a cotton swap soaked in normal saline and the end point was the appearance of fine punctuate bleeding (level of papillary dermis). Laser photoablation and gentile removal of photoablated tissue was repeated for 2-4 passes until the appearance of underlying pink tissue which was considered as the endpoint of laser photoablation. Postoperatively, patients were instructed to apply fucidic acid cream till crust formation and to keep the area clean and dry for the same duration. Photographic documentation of the lesion before laser treatment and immediately afterward and then reviewed at day 2, day 7, 1 month,2 months and finally at 6 months. Before and after photo were taken with Canon XUS 220 HS camera. 
The clinical response is graded by the treating physicians into four categories: excellent clearance (the skin of the lesion returned normal as the rest of the surrounding skin and does not need further treatment); good clearance ( $>75 \%$ cleared); moderate clearance $(>50$ - $75 \%$ cleared); and poor clearance $(<50 \%$ cleared).

Patients satisfaction questionnaire using a 4-point grading scale as follows: very satisfied, satisfied, somewhat satisfied and dissatisfied was completed by every patient at the end of follow up period. Any possible complications and side effects (erythema, texture changes, and scarring, post inflammatory pigmentations) were recorded during post-operative follow-ups.

Results

Fifteen patients (11 female and 4 male) with 23 clinically benignappearing melanocytic nevi completed the study. The mean age of the patients was 19.3 years (range 15-35). All included melanocytic nevi were involving the face. Among the sixteen AMN, 9 (39\%) were flat or just palpable pigmented lesion and categorized based on naked eye examination as junctional AMN while $7(30 \%)$ were dome-shape pigmented lesion and considered as compound AMN. While among the seven CMN, $6(26 \%)$ were smaller than $1.5 \mathrm{~cm}$ and categorized as small $\mathrm{CMN}$ and one $(4 \%)$ measured $2.3 \mathrm{~cm}$ which was considered as medium sized CMN (Figure 1).

\section{Figure 1: Clinical Types of The Studied Nevi}

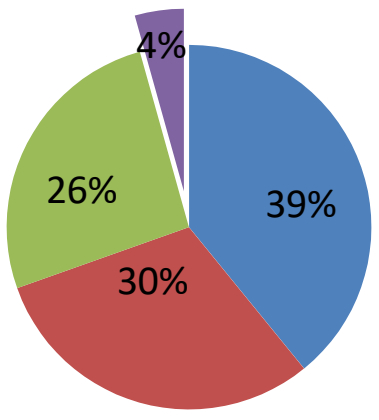

\section{Junctional}

AMN

Compound AMN

Figure 1:Seventy four treatment sessions were done using UCO2 laser and the number of treatments ranged from two to four sessions one month apart for every nevi (mean of 3.4 sessions). Two to four passes with UCO2 laser was undertaken in every laser session. Fiftytwo percent $(52 \%)$ of the included series showed good clearance of melanocytic nevi (clearance $>75 \%$ ) (Figures 2A and 2B) and $48 \%$ showed excellent results (Figures 3A - 4B) after two or four UCO2 laser sessions (Table 1).

\begin{tabular}{l|c|c|c|c|c|}
\hline \multirow{2}{*}{ Type of nevi } & Number & \multicolumn{4}{|c|}{ Clearance (\%) } \\
\cline { 3 - 6 } & & $\begin{array}{c}\text { Poor, } \\
\text { No. (\%) }\end{array}$ & $\begin{array}{c}\text { Moderate, } \\
\text { No. (\%) }\end{array}$ & $\begin{array}{c}\text { Good, No. } \\
(\%)\end{array}$ & $\begin{array}{c}\text { Excellent, } \\
\text { No. (\%) }\end{array}$ \\
\hline Junction & 9 & 0 & 0 & $3(33)$ & $6(67)$ \\
\hline Compound & 7 & 0 & 0 & $4(57)$ & $3(43)$ \\
\hline $\begin{array}{l}\text { Small } \\
\text { CMN }\end{array}$ & 6 & 0 & 0 & $4(67)$ & $2(33)$ \\
\hline $\begin{array}{l}\text { Medium } \\
\text { CMN }\end{array}$ & 1 & 0 & 0 & $1(100)$ & 0 \\
\hline Total & 23 & 0 & 0 & $12(52)$ & $11(48)$ \\
\hline
\end{tabular}
Table 1: Treatment
$\begin{aligned} & \text { Melanocytic Nevi after Treatment with Ultra pulsed CO2 Laser } \\
& \text { According to the Type of Nevus. }\end{aligned}$
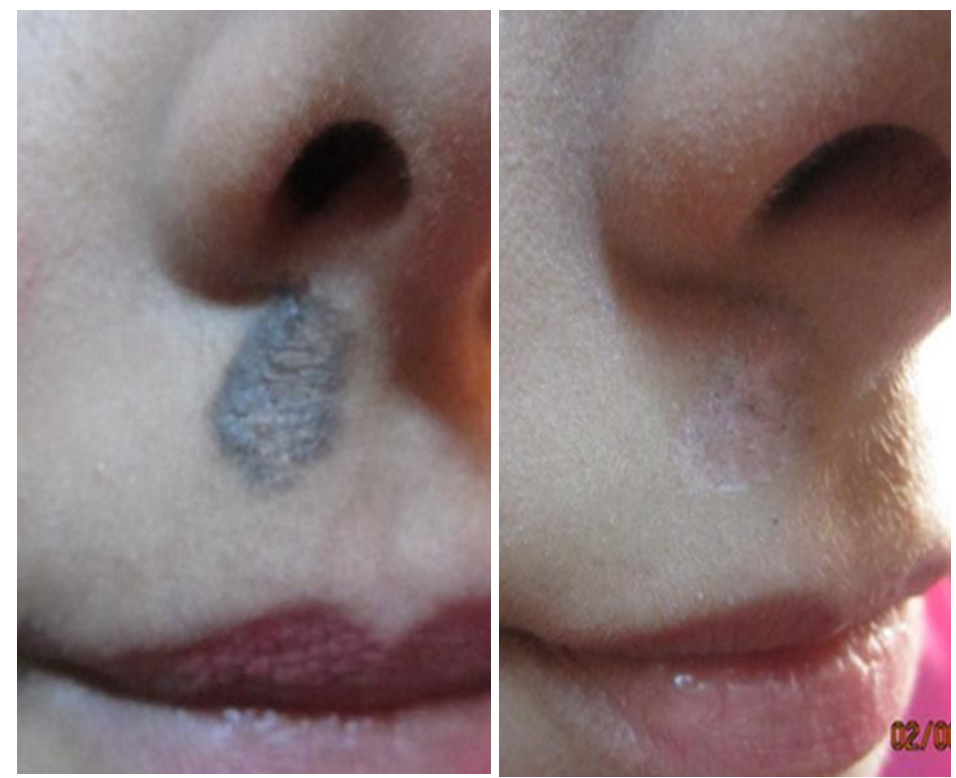

\section{(A: Before) (B: After)}

Figure 2: Fifteen years old female with medium-sized CMN just below the right nostril before (A) and after the fourth session of UCO2 laser treatment $(\mathrm{B})$ showing good clearance ( $>75 \%$ clearance).
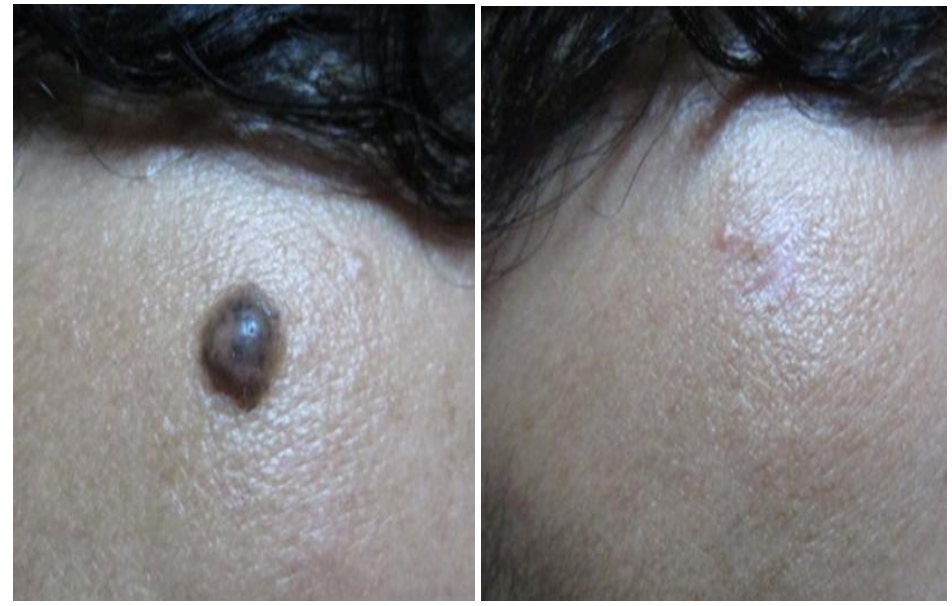

\section{(A: Before) (B: After)}

Figure 3: Twenty-two years old male patient with small $\mathrm{CMN}$ on forehead before (A) treatment and 6 months after second treatment session (B).

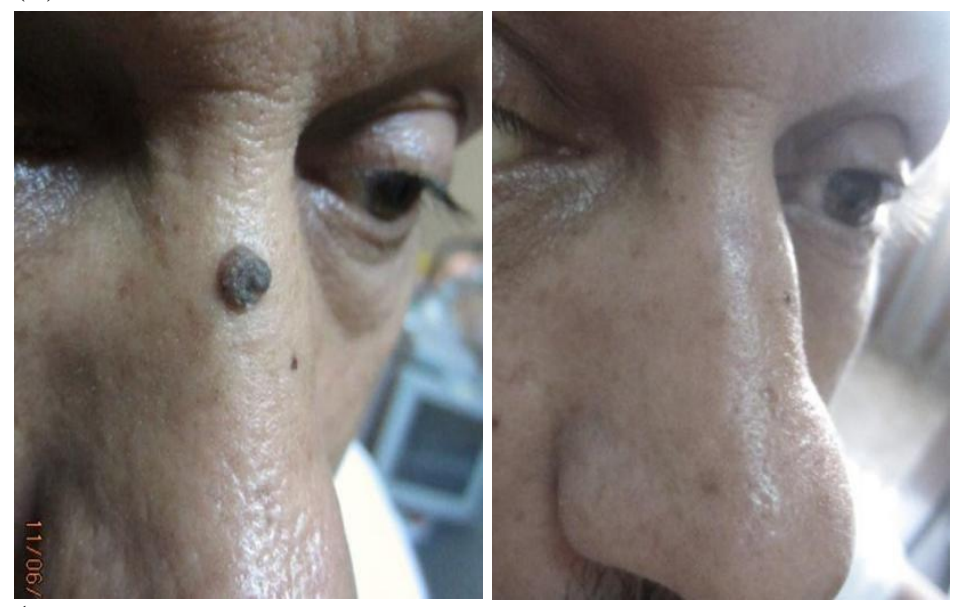

\section{(A: Before) (B: After)}

Figure 4: Thirty-five years old male with compound AMN on nose for more than ten years duration before(A) and 6 months after two laser sessions(B). 


\section{Figure 5: Overall Patients Satisfaction at The End of The Study}

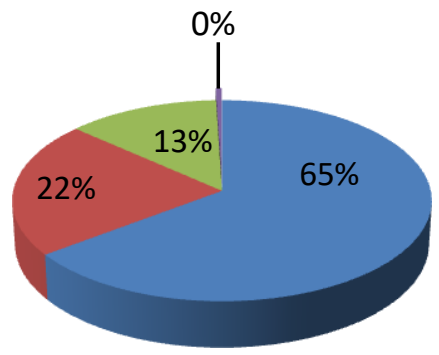

Very

Satisfied

Satisfied
Figure 5: Summarizes the assessment of patient satisfaction with the treatment results at the end of follow up period. Only one patient with small-sized CMN in this study showed mild textural changes during the post-operative follow period (Figure 6A and 6B).
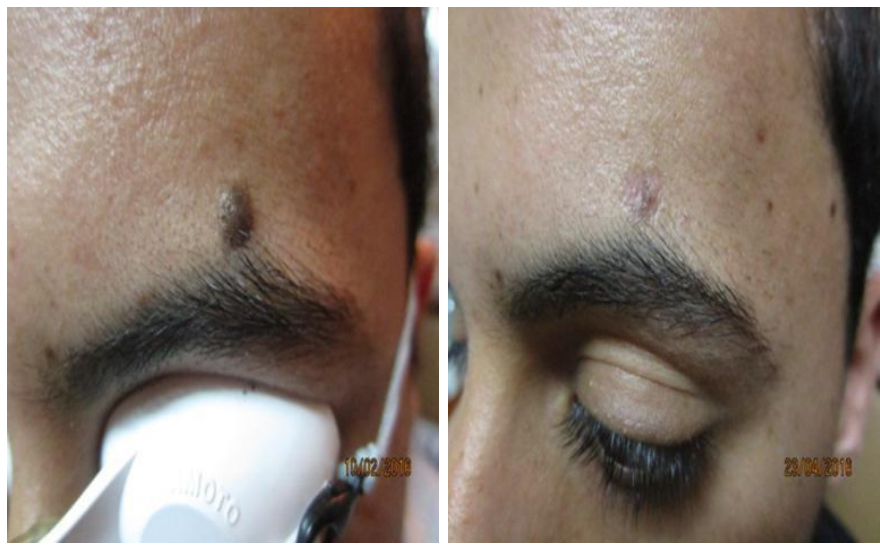

(A: Before) (B: After)

Figure 6: 16-year's old Libyan male patient with small-sized CMN above the left eyebrow before (A) treatment and 2- months after the second session of UCO2 laser treatment (B).

Discussion

Melanocytic nevi can be simply classified into congenital and acquired based on their first appearance since birth or thereafter. However, some CMN may not be obvious at birth but they gradually develop pigment over time and become apparent [2,4]. Generally, $\mathrm{CMN}$ are found to be much larger in diameter than acquired nevi. Majority of CMN are $5 \mathrm{~mm}$ or more in diameter at birth while most of AMN never grow to more than $5 \mathrm{~mm}$ [4]. AMN are benign proliferations of melanocytic naevus cells which occur initially within the epidermis at the dermo-epidermal junction hence called junctional melanocytic nevi. Further cellular proliferation results in the migration of nevus cells and their localization within the papillary dermis forming what is known as dermal melanocytic nevi. When the melanocytic naevus cells present in the dermo-epidermal junction and in the dermis, they are termed compound melanocytic nevi [5].

Various laser modality were used to treat melanocytic nevi including; pigment-selective ( $\mathrm{AL}, \mathrm{Nd}$ :YAG and fd Nd:YAG ), ablative lasers (Er:YAG and CO2) and combination of pigment-selective and ablating lasers; all have been found to be effective in the treatment of MN with variable outcomes and sequels. There are two types of pigment-selective laser the long-pulsed ( $\mathrm{msec}$ ) and short-pulsed (Qswitched nsec lasers) devices [5].
Most authors have agreed that the use of pigment-selective lasers either in the short-pulsed mode (Q-switched nsec) or in the long-pulsed mode $(\mathrm{msec})$ is effective in superficial nevi i.e. junctional nevi but with limited results in deeper lesions (compound and dermal nevus). Moreover, associated with high rate of recurrence $[5,10]$. This is because pigmentspecific lasers treat the superficial nevomelanocytes located in papillary dermis, but is unlikely to affect the deeply-seated nevomelanocytes. Which has been demonstrated histologically as persistent nevomelanocytic nests in the mid papillary and deeper dermis at depths of $0.16-0.44 \mathrm{~mm}$ following pigment-specific lasers sessions [7].

The principle of combination of pigment-selective and ablating lasers is to facilitate the delivery of laser beam of the pigment-selective lasers to reach to the deeply-seated nevomelanocytes after ablation of the superficial cells by the ablating lasers. This is in contrary to August et al. Who concluded that pretreatment of $\mathrm{CMN}$ with $\mathrm{CO} 2$ laser appears no more effective than pigment-specific laser alone and may increase the incidence of adverse effects $[7,8]$.

Ablating lasers (Er: YAG and $\mathrm{CO} 2$ ) work by destroying the target cells directly and the depth can be achieved by adjusting the dose. Er: YAG laser ablates nearly $5-50 \mu \mathrm{m}$ per pass while the UCO2 laser has a higher ablation depth of $20-60 \mu \mathrm{m}$ per pass and causes higher thermal coagulation which explain its complete destruction of nevus cells that minimize the risk of recurrence [5].

The overall results of our study pointed out that all included patients showed good to excellent results (Table1). This is come in accordance with Eggen et al. who recently systematically reviewed 24 studies with 20 different types of laser treatments in 434 patients and concluded that good to excellent result can be achieved in all laser categories (8). The best result in our series where accomplished from the AMN patients where $52 \%$ of patients showed good clearance (>75\% cleared) and $48 \%$ showed excellent results i.e. the skin returned to almost normal appearance. This promising outcome from our series is attributed obviously to the included AMN patients which comprise only junctional and compound nevi. The response of junctional and compound AMN is much better than dermal one, this is in consistence with many other studies $[5,7,8]$.

Regarding the studied six small size CMN, $(67 \%)$ showed good response and $(23 \%)$ showed excellent outcomes. The only one medium size CMN which measured $2.3 \mathrm{~cm}$ ended with complete clearance of the pigmentation but with mild textural changes though patient's satisfaction is very good.

The small number of included patients in our study is the major limitation. Furthermore, the lack of post-treatment histopathological report and the relatively short follow up period (6 months) represent another important drawback.

Malignant changes in nevi as a consequence to laser therapy remain of much worry and obstacle for practitioner in this field of dermatology but such burden have not been proved clinically [7]. Moreover recently, according to Eggen et al; treatment of melanocytic nevi with laser carry low risk of malignant transformation in contrary to the old assumption [8]. According to the author's opinion, this recent assumption will open the doors and will account for more liberal use of laser in this critical dermatological issue.

In conclusion, UCO2 laser is a marvelous device especially by expertise practitioner and represent a convenient substitute to the classical methods in the treatment of melanocytic nevus. It is a quick out-patient procedure, well tolerable by patient, need minimal postoperative care and associated with low risk of visible scarring. Moreover, a positive esthetic outcome. Therefore; we recommend the $\mathrm{UCO} 2$ laser for the treatment of melanocytic nevi but further studies with larger number of patients and longer duration of follow up is needed to prove the efficacy and safety of this procedure.

\section{References}

1. Atherton DJ (1998) Naevi and other developmental defects. In: Champion RH, Burton JL, Burns DA, Breathnach SM, (Eds.), Rook/Wilkinson/ Ebling Textbook of Dermatology, Volume 1. 6th Edition. London: Blackwell Science Limited; 519-616.

2. Marghoob AA (2002) Congenital melanocytic nevi: Evaluation and management. Dermatol Clin 20: 607-616 
3. Zayour M, Lazova R (2009) Congenital Melanocytic Nevi. Surgical Pathology Clinic 2: 457-469.

4. Senthilkumar M, Thappa DM (2006) Melanocytic nevi in children: A clinical study. Indian J Dermatol 51: 26-30.

5. Sardana K, Chakravarty P, Goel K (2014) Optimal management of common acquired melanocytic nevi (moles): current perspective. Clin, Cosm Invest Dermatol 7: 89-103.

6. Hong KT, Lee SE, Lim JM (2017) Treatment of Medium-toGiant Congenital Melanocytic Nevi with Combined Er:YAG Laser and Long-Pulsed Alexandrite Laser. Med Laser Sci 6(2): 77-85.

7. August PJ, Ferguson JE, Madan V (2011) A study of the efficacy of carbon dioxide and pigment-specific lasers in the treatment of medium sized congenital melanocytic naevi. Br J Dermatol 164: 1037-1042.

8.
Eggen CM, Lommerts JE, van Zuuren E, Limpens J, Pasmans SGMA, Wolkerstorfer A (2018) Laser treatment of congenital melanocytic naevi: A systematic Review. Br J Dermatol 178(2): 369383.

9. Pathania V, Chatterjee M (2015) Ultrapulse Carbon Dioxide Laser Ablation of Xanthelasma Palpebrarum: A Case Series. J Cutan Aesthet Surg 8(1): 46-49.

10. Suzuki H, Anderson RR (2005) Treatment of melanocytic nevi. Dermatol Ther 18(3): 217-226. 